\title{
Eine tiefe, frühe, neue Zeit. Anmerkungen zur hidden agenda der Frühneuzeitforschung
}

Arndt Brendecke

Die Frühe Neuzeit hat sich als Epochenbezeichnung akademisch und institutionell durchgesetzt, doch wer von der 'Geschichte der Frühen Neuzeit' außerhalb akademischer Selbstverständlichkeiten spricht, riskiert Fragezeichen: Was ist das, die frühe Zeit, einer neuen Zeit? Die Irritation wird hierbei nicht vom Zusatz 'früh' hervorgerufen, sondern im Grunde vom Begriff der 'Neuzeit' selbst. Das Wort mag zwar, wie schon Ferdinand Tönnies feststellte, „beinahe nichtssagend“ erscheinen. ${ }^{1}$ Doch als historiographisches Konzept ist es, insbesondere im deutschen Gebrauch, durch Widersprüche geprägt, ja geradezu damit aufgeladen, deren Konsequenzen für die hidden agenda der Frühneuzeitforschung hier diskutiert werden sollen. Meine These ist, dass diese Widersprüche wesentlich dafür verantwortlich sind, dass Frühneuzeitforschung in besonders intensiver Weise auf tiefenstrukturelle Interpretationen von Geschichte verpflichtet ist, weil sich nur auf diese Weise die Widersprüchlichkeiten auflösen lassen oder aber, bestimmten konzeptuellen Erwartungen - wie denen an die Neuzeitlichkeit der Neuzeit - begegnet werden kann. Der langen Tradition historiographischer Beschäftigung mit der Frühen Neuzeit hat dies keineswegs nur geschadet. Im Gegenteil: Es dürfte wesentlich für methodisch und darstellungstechnisch besonders innovative Leistungen verantwortlich sein.

Ich hebe zunächst die Widersprüche des Gebrauchs von 'Neuzeit' insbesondere in der deutschen historiographischen Tradition hervor (1). Daran anschließend gehe ich auf traditionelle historiographische Umgangsformen mit den Tiefenstrukturen der (frühen) Neuzeit ein (2), bevor zuletzt Vorschläge skizziert werden, wie sich diese Agenda aus dem Status ihrer schwachen Sichtbarkeit zu dem einer kontrollierten methodischen Programmatik entwickeln könnte (3).

1 Tönnies 1998, 17. 


\section{Konstitutive Widersprüche des historiographischen Konzeptes 'Neuzeit'}

Der Begriff 'Neuzeit' konnte sich in der deutschen Tradition nicht mit der gleichen positiven Emphase aufladen, wie sie der humanistischen Betonung von novitas oder dem kämpferischen Begriff der modernes in der französischen Querelle innewohnt und wie sie wenigstens anteilsmäßig noch in Begriffen wie histoire moderne, modern history usw. enthalten ist. ${ }^{2}$ Dies hat u. a. mit einer Reihe von geradezu konstitutiven Widersprüchen zu tun, die sich in die Bedeutung des deutschen Neuzeitbegriffs eingeschrieben haben.

Unter konfessionellen Gesichtspunkten erwies sich die Betonung der novitas in Deutschland über lange Zeit hinweg als nur beschränkt sinnvoll, und zwar nicht nur für die katholische Historiographie, sondern gerade auch für die protestantische. Luther bezeichnete seine Gegenwart als eine 'alte Zeit' („Nos iam sumus in senectute“) ${ }^{3}$. Dass später dann protestantische Autoren die Formel einer historia nova etablierten, ist insofern paradox, als eine solche Zeitspanne aus der Perspektive der reformatorischen Parusie-Erwartung keinerlei geschichtstheologischen Sinn machte, ja eigentlich die Reformation sogar entwertete. Dennoch wuchs die Notwendigkeit, diesem "großen, sich bis in die Gegenwart fortsetzenden Kontinuum" (Stephan Skalweit) ${ }^{4}$ einen Namen zu geben, wobei die Semantik der 'Neuheit' den Vorteil mit sich brachte, damit die Differenz zum papstkirchlichen Mittelalter markieren zu können. Schon Christoph Cellarius nannte so in seiner Historia nova von 1702 nicht mehr nur die Eroberung Konstantinopels als Markstein einer Epochengrenze, sondern auch die Reformation. ${ }^{5}$

Im 19. Jahrhundert waren es erneut vorwiegend protestantische Historiker, die den Begriff mit Bedeutung füllten. Dies geschah dann bereits mit einer auf die Kategorien 'Staat' und 'Nation' ausgerichteten Wertematrix, die gewagte Interpretationen der Frühen Neuzeit notwendig

2 Zum Epochenbewusstsein in Deutschland um 1500: Müller 1991, 121-144; dagegen zur in Deutschland vergleichsweise schwachen Ausprägung des Gedankens einer Wiederbelebung oder Wiedergeburt der Antike um 1500: Worstbrock 1974, 506.

3 Luther 1912-1921, Nr. 435, 188.

4 Skalweit 1987, $227 \mathrm{f}$.

5 „In primis Ecclesiae reformatio meretur, ut novam historiam, distinctam ab illa quae medii aevi fuit, ex saeculo decimo sexto, aut prope illius initia, auspicemur" (Cellarius 1696, 3). 
machte, stand diese Epoche doch in Hinsicht auf beide Kategorien im Zeichen eines Defizits. Der Frühen Neuzeit schien ausgerechnet das entscheidend Neue - 'Nation' und 'Staatlichkeit' - markant zu fehlen, ja es gehörte geradezu zu den Qualitäten des Alten Reiches, in Hinsicht auf diese Kategorien schwach zu sein. ${ }^{6}$

Insgesamt kann man sagen, dass sich zwar auch in der deutschen Geschichtsschreibung so schon früh schulbuchmäßig einzelne Marksteine für 'Neuzeit' aufzählen ließen. Doch die semantische Suggestion des Neuzeitbegriffes von 'etwas Neuem' steht traditionellerweise in einem starken Widerspruch zu den privilegierten historischen Inhalten. Die deutsche Reformation trat früher in die Weltgeschichte ein, als sie es von sich selbst heilsgeschichtlich behauptete, Staat und Nation kamen hingegen reichlich spät. Die Emphase der 'Neuheit' musste der deutschen Geschichtswissenschaft des 19. Jahrhunderts - und nicht nur dieser $-^{7}$ in Hinsicht auf alle ihre Leitkategorien im Halse stecken bleiben, schon aufgrund der skizzierten Widersprüche, aber auch, weil wenigstens die 'Reformation' und 'Nation' als historische Gestaltwerdung von etwas sehr, sehr Altem verstanden wurden. ${ }^{8}$

Interessanterweise haben sich der Begriff und das historiographische Konzept der 'Neuzeit' dennoch genau in dieser Phase des späteren 19. Jahrhunderts durchgesetzt, wenngleich Kosellecks lange Zeit geltender Befund, das Wort 'Neuzeit' habe erst um 1870 überhaupt in die deutsche Sprache Eingang gefunden, inzwischen (auch von ihm selbst) korrigiert wurde. 'Schon seit den 1840er-Jahren häufen sich Publikationen, die das Wort 'Neuzeit' im Titel führen. ${ }^{10}$ Hier muss jedoch nicht die Begriffsgeschichte verfolgt werden. Es hat zu interessieren, welche Folgen die enorme Spreizung zwischen der Betonung von novitas als dem entscheidenden Epochenkennzeichen und dem inhaltlichen Befund vergleichsweise später,

6 Wolfgang Menzel, allerdings kein typischer Vertreter des Protestantismus, verstand beispielsweise als das ,großartigste und welthistorisch wichtigste [Werk] der ganzen Neuzeit, [...] die Wiedervereinigung der so lange unnatürlich getrennten deutschen Volksstämme“, vgl. Menzel 1871, 1.

7 Auch der auf Deutschland spezialisierte, englische Mediävist Geoffrey Barraclough stellte fest: „After all, what is by now three hundred or four hundred years old is only 'modern' by courtesy." Zit. nach Pot 1999, 324.

$8 \mathrm{Zu}$ diesen und anderen Paradoxien des Frühneuzeit- und Modernekonzeptes: Reinhard 1993, sowie Goldstone 1998; bezüglich des Mittelalters: Rexroth 2008, $12-31$.

9 Vgl. Koselleck 1979, 302 f. Koselleck korrigierte sich selbst in: ders. 2003, 227.

10 Z.B.: Marggraff 1847; Hoffmann 1848. 
nur langsam sich durchsetzender Innovativität nach sich zog, denn diese Spannung betrifft in besonderem Maße die frühe Neuzeit.

Meine These ist, dass diese Spannung u. a. dazu führte, dass man bei der Erforschung und Interpretation der Geschichte der Frühen Neuzeit traditionell stärker am Invisiblen und Latenten interessiert ist, an Unterströmen, deren Verlauf, Stärke und Richtung sich durch eine einfache Lektüre der Quellen nicht gleich erschließen. Ohne Zweifel hat dies ambivalente Folgen. Es ermutigt einerseits zu gewagten Unterstellungen bezüglich unsichtbarer Wesentlichkeiten der Frühen Neuzeit (ihrem 'Geist', ihrer 'Gestalt' usw.). Es trieb aber immer wieder auch an, um mit geduldiger, präziser Quellenarbeit und einer darauf zugespitzten Methode Randphänomene und Unterströmungen dieses Zeitraumes heraus zu präparieren. Während das eine nach Verallgemeinerungen strebt, sucht das andere nach Besonderheiten. So innovativ beides sein kann, so sehr verlangt es doch auch in einem besonders intensiven Maße nach Abstimmung, ja nach reflektierter Verzahnung. Geschichte der Frühen Neuzeit zu schreiben, geht jedenfalls von Anfang an mit dieser problematisch-kreativen Herausforderung einher. Sie ist es, welche die schon seit längerer Zeit etablierte hidden agenda der Frühneuzeitforschung wesentlich bestimmt.

\section{Die Invisibilität des Wesentlichen. Traditionelle Tiefenstrukturen der Frühneuzeitforschung}

Die Frage der Epochenabgrenzung ist mit noch so guten Vorschlägen für Marksteine in der Chronologie nicht zu beantworten. Schon bei Leopold von Ranke findet sich die Klausel, dass die üblichen Epochenmarker bloß symbolhaft für tiefer liegende und langwierigere Veränderungen stehen würden. ${ }^{11}$ Reinhart Koselleck hat-die methodische Konsequenz ziehend-betont, dass Epochenabgrenzung wesentlich eine Frage der Tiefenstruktur von Geschichte ist. ${ }^{12}$ Bevor keine Einigung über die zu beobachtende 'Schicht' erzielt wird, lässt sich das historische Kontinuum nicht überzeugend skandieren. Da somit auch die Rolle der Frühen Neuzeit im Epochenverlauf von Entscheidungen über die präferierte historische Tiefenstruktur abhängt, ist das Problem hier aufzugreifen.

Ziemlich klar zeigt sich, dass der Epochenbruch umso früher liegt, je mehr von den Historikern auf markante Einzelerscheinungen fokussiert

11 Vgl. Ranke 1975, 104 f.

12 Vgl. Koselleck 2003, 238 f. 
wird. Wo man bevorzugt auf besondere Taten, Menschen oder Ideen blickt, lässt sich eine Pionierlinie erster 'Taten' und 'Gedanken' zeichnen, die je nach Sektor irgendwo zwischen dem 12. und 16. Jahrhundert, bevorzugt aber in der Zeit des Humanismus liegt. Dieser Modus einer Herausarbeitung des Bruches über Verweise auf Pionierleistungen ist besonders deutlich (und pathetisch) in Lord Actons Antrittsvorlesung von 1895 aufzufinden:

I describe as Modern History that which begins four hundred years ago, which is marked off by an evident and intelligible line from the time immediately preceding, and displays in its course specific and distinctive characteristics of its own. The modern age did not proceed from the medieval by normal succession, with outward tokes of legitimate descent. Unheralded, it found a new order of things, under a law of innovation, sapping the ancient reign of continuity. In those days Columbus subverted the notions of the world, and reversed the conditions of production, wealth, and power; in those days Machiavelli released government from the restraint of law; Erasmus diverted the current of ancient learning from profane into Christian channels; Luther broke the chain of authority and tradition at the strongest link; [...]. The like effects are visible everywhere, and one generation beheld them all. It was an awakening of new life; the world revolved in a different orbit. ${ }^{13}$

Fokussiert man indes auf die Ebene gesellschaftsdurchdringender Konsequenzen, dann scheint Modernität nicht am Anfang, sondern am Ende der Frühen Neuzeit zu liegen, oft schlicht deshalb, weil sich deren Breitenwirkung erst zeitlich verzögert feststellen lässt. Diese Grundregel gilt für viele Bereiche, etwa für den der Religionsgeschichte. Im Pioniermodus kann man mit Adolf von Harnack über die Neuzeit behaupten: „die Hammerschläge an die Türe der Schlosskirche zu Wittenberg haben sie eingeleitet ${ }^{"} .{ }^{14}$ Fragt man jedoch nach tiefergehenden religionsgeschichtlichen Effekten, so lässt sich der Protestantismus des 16. und 17. Jahrhunderts-etwa mit den Argumenten Ernst Troeltschs-auch als Fortsetzung mittelalterlichen Geistes verstehen. Erst die Aufklärung würde dann den entscheidenden Bruch markieren. ${ }^{15}$ Bedeutet dies, dass die Frühe Neuzeit je nach Betrachtungstiefe entweder noch vormodern oder schon

13 John Emerich Edward Dalberg Acton, zitiert nach Pot 1999, $321 \mathrm{f}$.

14 Zitiert nach Pot 1999, 321.

15 Vgl. Schorn-Schütte 1999a, 49. Troeltsch unterscheidet jedoch an anderer Stelle zwischen einer Neuzeit im weiteren und im engeren Sinne: „Im ersteren [weiteren] Sinne beginnt sie mit dem 15. Jahrhundert und dem neuen Militär- und Beamtenstaat der Souveränität gegen Kirche und gegen Imperium, im zweiten [engeren] Sinne beginnt sie mit der englischen Revolution und der Aufklärung"; zitiert nach Pot $1999,323 \mathrm{f}$. 
frühmodern ist? ${ }^{16}$ Dass sie also sowohl vor wie auch nach dem großen Bruch platzierbar ist?

Der Fall liegt komplizierter, denn es gibt eine lange Tradition historiographischer Techniken, den Mangel an Breite und Sichtbarkeit eines frühen Phänomens zu kompensieren. Georg Wilhelm Friedrich Hegel wies dazu den Weg, indem er beispielsweise postulierte, dass mit Luthers emphatischem Gewissensbegriff das Prinzip der Subjektivität welthistorisch zu tragen begann, auf dessen Grundlage sich schließlich Freiheit Bahn brach und die Staaten beginnen konnten, die Gesetze der Freiheit zu realisieren. Wie Philippe Büttgen herausgearbeitet hat, liegt die Besonderheit dieser Konstruktion gerade in der Unsichtbarkeit des Epochemachenden. ${ }^{17}$ Denn in der lutherischen Tradition wird das Gewissen über seine Unverfügbarkeit gegenüber allem Äußeren konstituiert, sodass dieses für Hegel welthistorisch so entscheidende Potential überhaupt erst durch Innerlichkeit realisiert werden konnte. Die Invisibilität des Phänomens ist im Sinne dieser Interpretation also kein Argument für die Herabstufung seiner historischen Bedeutung. Ganz im Gegenteil: Das Entscheidende ist notwendigerweise verhüllt, es entzieht sich dem Blick, ist 'Geist', und als solcher besonders wirkmächtig.

Wie auch immer man über diese Interpretation inhaltlich denken mag: Nicht nur Hegels Wertsetzungen, sondern auch seine Technik stufenweiser Sichtbar- bzw. Bewusstwerdung des historisch Wesentlichen besaß eine hohe Attraktivität zuerst für die protestantisch dominierte deutschsprachige Geschichtswissenschaft des 19. Jahrhunderts und dann wohl auch wenngleich dies einer intensiveren Überprüfung bedürfte - der entsprechenden historischen Forschung der Zwischen- und Nachkriegszeit. Im 19. Jahrhundert finden sich - etwa in Rankes hierbei klar auf Hegel Bezug nehmenden Vorlesungen - die Hinweise auf die entscheidende Bedeutung des „inneren Entwicklungsganges“ allenthalben wie auch Statements, mit denen man der Hörerschaft das Festhalten der älteren Historiographie an „allem Äußerlichen“ als Problem vor Augen führte, das Herausarbeiten der "geistigen Potenzen“ und „Ideen“ als Lösung. ${ }^{18}$ In der Zwischenkriegszeit schließlich, d.h. noch vor der institutionellen Etablierung der Frühen Neuzeit, entstanden eine Reihe großer Thesen und Studien über den formativen, meist unsichtbaren Wandel der frühen Neuzeit. ${ }^{19}$ Gerade bei

16 Dazu in anderer Perspektive: Zwierlein 2009.

17 Vgl. Büttgen 2009.

18 Ranke 1975, 138 f., 185, 191.

19 Zur Zwischenkriegszeit wegweisend: Schorn-Schütte 1999b. 
den deutschen Autoren geschah dies häufig mit expliziter Nennung des zeitgenössischen Leidensdruckes, etwa im Falle von Willy Andreas' Studie Die große Wende von 1932. Dort wird betont, in welchem Maße „die ungeheuren Erlebnisse des Ersten Weltkrieges und des Zusammenbruchs, [...] uns mit all ihren Folgeerscheinungen die tiefe Fragwürdigkeit des Lebens und aller menschlichen Einrichtungen gelehrt haben" ${ }^{20}$ Zugleich wird konstatiert, dass aus diesen Erschütterungen eine besondere heuristische Kompetenz hervorgegangen sei. Sie erlaube es, so die Worte von Willy Andreas, „aus der Krisenhaftigkeit der eigenen wie der allgemeinen Existenz heraus vergangenes Lebens bis in seine gefährlichsten Tiefen hinein nachzufühlen, und namentlich dann, wenn es in gleich stürmischer Wallung wie das unsere dahinbraust. ${ }^{\text {"21 }}$ Allenthalben wurde nach tieferen historischen Ursachen für die Erschütterung der Werte schon um 1900 und dann für die Katastrophen der ersten Hälfte des 20. Jahrhunderts gesucht, wozu nun die (später sogenannte) Frühe Neuzeit das entscheidende Untersuchungsfeld darstellte. Auf diesem Feld ließ sich etwa die folgende Frage Gerhard Oestreichs bezüglich des 'Preußentums' traktieren: „Woher kam nun diese einmalige Energie, welcher Art sind die geistigen Werte, die hinter der betont erzieherischen Arbeit der brandenburg-preußischen Dynastie stehen?" 22 Im Falle Oestreichs trieben solche Fragen dazu, neue methodische Maßstäbe in der Kombinierung von Verfassungs- und Ideengeschichte zu setzen, gleichsam die äußere und innere Bedingtheit einer spezifischen Haltung der Moderne in den Blick zu bekommen. Dennoch: Auch bei Oestreich bleiben die historisch entscheidenden Triebkräfte (Neostoizismus, Disziplinierungseffekte usw.) invisibel.

Die erfahrungsgetriebene Suche nach Tiefe, schicksalhafter, ja „stürmischster" Bewegung dieser Historikergenerationen setzte Geschichte nicht nur heuristisch unter Kreativitätsdruck, sondern auch darstellungstechnisch. Wie darauf reagiert werden konnte, zeigen schlaglichtartig Geschichtsfilme der 1920er bis 1960er Jahre: bedeutungsschwere Bilder und Sätze, Protagonisten, die im Moment der Entscheidung äußerlich stillstehen, weil nur auf diese Weise die eigentliche, innere Bewegung 'gezeigt' werden kann. ${ }^{23}$ So schwer dieses innerliche Ringen - die innere Handlung - darstellbar ist, so unersetzlich sind die Techniken der Ruhigstellung der Umgebung und des Aussprechens von Schlüsselsätzen wie

20 Andreas [ $\left.{ }^{6} 1932\right] 1959,9$.

21 Andreas [ $\left.{ }^{6} 1932\right] 1959,9$.

22 Oestreich 1989, $157 \mathrm{f}$.

23 Zum Phänomen bereits Kracauer 1993, 104. 
Luthers „Hier stehe ich, und kann nicht anders“, der bezeichnenderweise nicht eine Handlung betont, sondern eine - im Gewissen wurzelnde Handlungsblockade. ${ }^{24}$ Wieder sehen wir, wie das Entscheidende invisibel bleibt und sich daraus sowohl heuristische wie darstellungstechnische Herausforderungen ergeben. Sie haben sich auch für die wissenschaftliche Arbeit an Geschichte keineswegs - etwa im Zuge der 'Krise des Historismus' - erledigt. Im Gegenteil: Die heute in den Sozialwissenschaften präferierte (und gerne überhöhte) Grenze zwischen Vormoderne und Moderne wird weiter über die Veränderung einer inneren Haltung definiert. Bei Peter Wagner heißt es beispielsweise: „Überall dort, wo Menschen sich selbst als autonome Wesen begreifen, ist 'Moderne' ${ }^{\text {'“ }}{ }^{25}$ Innerhalb der Fachwissenschaft wurde darauf methodisch sensibler reagiert, unter anderem durch eine spürbare Präferenz für Ideengeschichte und für Prozessbegriffe, welche die historisch maßgebliche Bewegung nicht mehr nur ins Innerste einzelner Pioniere - wie Martin Luther - verlegten, sondern nun in demokratisierter Weise das Innerste des frühneuzeitlichen Menschen an sich thematisierten ('Geist des Kapitalismus', Sozialdisziplinierung, Mentalitätsgeschichte, Konfessionalisierung usw.).

Es würde eine eigene Untersuchung erfordern, nachzuweisen, in welchem Maße die Frühneuzeitgeschichte über die Phasen der Herausbildung dieses Themenfeldes und dann der akademischen Institutionalisierung in das Spiel solcher, auf das Verborgene abzielenden Umbruchsbehauptungen verstrickt war. Dass diejenige Generation bundesrepublikanischer Historiker, welche schließlich die Frühneuzeitforschung akademisch mit begründete, die Epoche als „Inkubationszeit der Moderne“ (Paul Münch) oder als deren „Musterbuch“ (Winfried Schulze) bezeichnete, trägt insofern eine doppelte Wahrheit in sich, die jedoch nur zur Hälfte ausgesprochen wurde. ${ }^{26}$ Denn Frühneuzeitforschung ist nicht nur inhaltlich an Vorformen der Moderne interessiert, die man auf diese oder jene Weise herausarbeiten und diskutieren sollte. Ihr ganzer Arbeitsmodus, ihre Heuristik, ihre Darstellungsweise ist am Modell der Inkubation geschult. Über das dem Fach Geschichte eigentümliche Maß hinaus steht Frühneuzeitforschung deshalb in der Tradition, gerade nicht bloß Fakten abzusichern. Die Grundannahme der Verborgenheit entscheidender Wand-

24 Vgl. etwa die Darstellung dieser Szene durch Niall MacGinnis als Luther in dem deutsch-amerikanischen Spielfilm Martin Luther von 1953.

25 Wagner 2009, 17; zu den Unterscheidungen Vormoderne/Moderne nun zusammenfassend: Zwierlein 2011.

26 Vgl. Münch 1984, 15; zu weiteren Stellen und darüber hinaus: Schulze 1993. 
lungsprozesse fordert vielmehr permanent dazu heraus, mit großer Findigkeit Methoden der Sichtbarmachung und narrativen Aufwertung des Latenten einzusetzen, was die hohe Innovationsfreudigkeit der Frühneuzeitforschung mit erklärt, ihre Pionierleistungen etwa auf dem Gebiet der Mentalitäts-, Wahrnehmungs- und Ideengeschichte oder der Kultur- und Frömmigkeitsforschung. ${ }^{27}$

Es ist im Rahmen eines Aufsatzes nicht möglich, die methodische Problematik einer solchen historiographischen Tradition der Frühneuzeitforschung als einer 'tiefen', 'frühen', 'neuen' Zeit sowie die dadurch bedingten interpretatorischen Effekte wirklich herauszuarbeiten. Im Sinne der Revision einer Epoche scheint es mir jedoch notwendig, wenigstens skizzenartig auf die mögliche zukünftige Gestaltung der Agenda der Frühneuzeitforschung einzugehen.

\section{Revision der Tiefe. Zur zukünftigen Gestaltung der Agenda der Frühneuzeitforschung}

Hervorzuheben ist zunächst nochmals, dass Frühneuzeitforschung nicht zufällig eine besondere Tradition des Interesses an tiefen, verborgenen Schichten des Historischen vorweisen kann. Diese Prädisposition ist wesentlich ihrer chronologischen Stellung im Vorfeld der Moderne geschuldet. Denn die verschiedenen Projekte, Prägungen und Brucherfahrungen der Moderne trieben Fragen hervor, welche sich schlechterdings nur über eine Verlegung von entscheidenden Teilen des Untersuchungsfeldes zugleich ins historische Vorfeld und in die Tiefe beantworten (wie auch teilweise sistieren) ließen. Im Ergebnis stehen am Anfang der frühen Neuzeit verborgene Umbrüche - die hidden revolutions der kopernikanischen Wende, des Gewissens, der Individualität, des Humanismus, der Konfessionalisierung usw., während das Ende der Frühen Neuzeit und die eigentliche Moderne viel stärker durch nach außen gewendete, wesentlich materieller auftretende Revolutionen des Politischen, der Ökonomie, der Industrialisierung und der sozialen Lebenswelten markiert wird. Zwei Brüche also, die Veränderung in verschiedenen Tiefen ausloten. Für den zweiten Bruch ('um 1800') ist, insbesondere durch die Arbeiten Kosellecks eine intensive Beschäftigung mit dem schichtartigen Aufbau des Wandlungsprozesses und den Interdependenzen zwischen den Schichten (d.h.

27 Zur Frage nach der methodischen Zugänglichkeit des ebenso Geschichtsmächtigen wie Unsichtbaren auch Tschopp 2005. 
der Veränderung der Begriffe, der Zeitkonzepte, des politischen Systems, der Öffentlichkeit, der Wirtschaftsform usw.) ausgelöst worden, während sich zugleich der erste Bruch ('um 1500') zunehmend aufzulösen scheint. Bedauerlich erscheint mir dies nur in Hinsicht auf zwei Konsequenzen, nämlich erstens einer (meist unausgesprochenen) Präferenz für relativ oberflächliche Merkmale des historischen Wandels wie das der Veränderung von politischen Systemen und Diskursen. Zweitens ist zu beklagen, dass im Zuge der Überhöhung des Bruches 'um 1800' die Frühe Neuzeit für die historische Erklärung der Moderne an Bedeutung verlor. Zwar ist weder das übermäßige Interesse an der Bestimmung des Bruches selbst noch die Tradition hegelianischer Unterstellungen unsichtbarer Wesentlichkeit zurückzuwünschen. Sehr wohl gilt es aber, sich sowohl die Möglichkeit methodisch tiefgreifender (vertikaler, d.h. mehrere Schichten durchdringender und verknüpfender) Analyse zu erhalten wie auch jene, längerfristige historische Kontinuitäten in den Blick zu nehmen und in die historischen Modelle zu integrieren.

Die Chancen dafür könnten besser nicht sein: Die Moderne setzt mit abnehmender Selbstverständlichkeit die Kategorien, mit der wir das historische Feld organisieren. ${ }^{28}$ Ihre Erfahrungen und Kategorien gleiten stückweise selbst in die Vergangenheit, sodass es die Moderne selbst ist, die nun zu historisieren ist. Das ist ein langwieriges Vorhaben, welches jedoch die explanatorische Rolle der Frühen Neuzeit neu definiert, der weiter eine - allerdings gewandelte - Schlüsselrolle zufällt. War sie seit den 1970erJahren in die Aufgabe verstrickt, die Genese der Moderne zu erklären, also zu zeigen, wann und in welcher Weise sich Modernität Bahn brach, auf welche Widerstände oder Lösungen sie dabei stieß, so könnte sie nun einer kritischen Historisierung der Moderne zuarbeiten, d. h. einer solchen, die nicht von den Leistungen und Versprechungen der Moderne gebannt ist, sondern sich zur Aufgabe nimmt, die Geschichte ihrer Doppelbödigkeiten und Unabgeschlossenheiten, ihrer Asymmetrien zu erschließen, solcher Strukturen also, die die Moderne, ja eine sehr lange Moderne, eben auch kennzeichnen, obschon nicht in gleichartig plakativer Weise. Damit verschiebt sich das Verhältnis der Frühneuzeit zur Moderne. Die Frühe Neuzeit steht nicht mehr in einem - man könnte sagen - historisch affirmativen Begründungsverhältnis zur Moderne, sie hat nicht mehr die historiographische Bringschuld der Moderne zu tragen, ihre Fragen zu beantworten. Im Gegenteil: Sie trägt nun das Potenzial in sich, zu einer kritischen Historisierung der Moderne beizutragen. Von ihrem Stand-

28 Dazu bereits, wenngleich mit anderen Konsequenzen: Schulze 1990, $84 \mathrm{f}$. 
punkt aus, so die These, lassen sich privilegierte Einsichten in die verdeckten, aber nach wie vor tragenden Fundamente einer langen Gegenwart erwarten. Damit das gelingen kann, muss sorgfältig zwischen den positiven Effekten einer tiefenstrukturierten Betrachtung von Geschichte einerseits und den Beschränkungen der verschiedenen Modi, dies zu tun, andererseits abgewogen werden. Es geht also um Bewusstmachung unseres historiographischen Umgangs mit den Tiefenstrukturen, um die Kontrolle des Verfahrens. Dazu gehört es selbstverständlich auch, die eingespielten Narrative einer neuerlichen Revision zu unterziehen, was von der Arbeit Hayden Whites bereits gefordert - explizit auch in Hinsicht auf die 'Tiefenstrukturen' -, aber keineswegs hinreichend geleistet wurde. Anknüpfen lässt sich hier an Modelle der Annales-Schule und an eine große Zahl bereits geleisteter Mikro- und Detailstudien, deren an sich so begrüßenswerte Vielfalt jedoch verstärkt durch Versuche zu begleiten wäre, von beliebigen crossovers zu kontrollierten Vertikalanalysen zu gelangen, die ein Thema in der Brechung aller relevanten Schichten durcharbeiten.

Für eine in diesem Sinne zu leistende, methodisch reflektierte Weiterentwicklung der Agenda der Frühneuzeitforschung wäre es meines Erachtens notwendig, sich erstens mit dem Plot der Moderne auseinanderzusetzen, der unser Design nach wie vor über die Maßen bestimmt. Zweitens sollten wir unsere Beobachtungspräferenz von den Postulaten einer Zeit auf deren meist latente Praktiken verschieben.

Mit dem Plot der Moderne meine ich Routinen der Benennung, Beschreibung und Erklärung, die - man könnte sagen - eine strukturelle Teleologie in sich tragen, weil sie stets Varianten einer einzigen Erzählung provozieren, nämlich derjenigen der Überwindung des Status Quo. ${ }^{29}$ Diese Routinen haben sich längst in die Begriffe eingeschrieben, mit deren Hilfe wir Heuristik und Explanatorik organisieren. Ganz gleich ob wir beispielsweise mit eher statischen Epochenbegriffen wie 'Konfessionelles Zeitalter', 'Absolutismus' und 'Gegenreformation' oder mit Prozessbegriffen arbeiten, wie 'Konfessionalisierung', 'Säkularisierung' oder 'Sozialdisziplinierung', drängen wir damit die Realien unseres Untersuchungszeitraums in den Dialog mit einem mächtigen, die Epoche

29 Monumental ist dieser Plot in Arnold Toynbees A Study of History zur Anwendung gekommen, in der die Geschichte der Zivilisationen über die Spannung zwischen challenges und responses strukturiert wird. Damit schafft es Toynbee, Weltgeschichte jenseits deterministischer Schicksalhaftigkeiten des Auf- und Untergangs der Völker zu dramatisieren, jedoch um den Preis der Festlegung auf einen dramatischen Plot. 
überspannenden, ja im Grunde immer über deren Zeit hinausweisenden Idealtypus. Das mag helfen, bestimmte Tendenzen einer Epoche hervorzuheben, jedoch um den Preis einer übermächtigen Polung auf etwas, das jenseits der Epoche, meist sogar außerhalb des historisch Gegebenen an sich liegt (denn nie gab es eine gänzlich konfessionalisierte Gesellschaft, nie einen vollständig absoluten Herrscher usw.).

Auch Max Webers Idealtyp gehört damit auf den Prüfstand. Wie Weber selbst hervorhob, ist der Idealtyp ein Hilfsmittel mit doppeltem Zweck. Erstens soll damit auf eine möglichst reflektierte Weise zwischen dem empirisch Gegebenen und der Welt der Begriffe vermittelt werden. Zweitens aber geht er über die Funktion begrifflicher Abbildung von Realität hinaus. Er dient dazu, soziale oder historische Tatbestände unter dem Gesichtspunkt ihrer Kulturbedeutung untersuchen zu können. Begrifflich idealisiert wird also nicht bloß zum Zwecke der Komplexitätsreduktion und Verallgemeinerung, sondern immer auch, um Einzelerscheinungen über das Instrument der idealtypischen Messung zu bewerten.

Problematisch wird es nun, wenn historisches Arbeiten über idealtypisch konstruierte Epochen- und Prozessbegriffe organisiert wird. Das Feld der Streuung realer Phänomene wird in einer Weise vom Idealtypus beherrscht, die (verdeckt) wertet, indem sie Nähe zum Ideal prämiert. Mit Max Webers Worten: Man hat deren „typologischen Ort durch Ermittlung der Nähe oder des Abstands vom theoretisch konstruierten Typus festzustellen “. ${ }^{30}$ Ein Teil der Operation mag unvermeidlich sein, wo immer man überhaupt verallgemeinernde Begriffe setzt. Doch es gibt durchaus Alternativen zur Idealisierung der 'Mitte' des Streufeldes, beispielsweise Begriffe, die sich stärker auf die Ausdehnung, Gestalt und Veränderung dieses Feldes oder auf seine Ränder konzentrieren. Mehr noch: Historiker untersuchen vorwiegend Veränderungen durch die Zeit, sie verknüpfen deshalb ihre wichtigsten Begriffe fast notwendigerweise mit der Zeitachse. Wenn diese Begriffe dann idealtypisch konstruiert wurden, - etwa um eine bestimmte historische Entwicklung (der Säkularisierung, der Konfessionalisierung usw.) herausarbeiten zu können, setzt dies einen Bewertungsmechanismus in Gang und zugleich den Plot der Moderne ins Werk. Denn dann fallen das Fortschreiten in der Zeit und die Annäherung an das Ziel in eins, Geschichte ist dann Fortschritt, Gegenphänomene stehen für Stagnation oder Widerstände. Mächtiger kann eine Teleologie nicht sein. Wer auf diese Weise 'Säkularisierung' sagt, prämiert Phänomene in Bewegung von der Religion zum Säkularen. Wer 'Sozialdisziplinierung' sagt,

30 Weber 1920, 537. 
betrachtet Akteure auf einem Stück des Weges zu einer disziplinierten Gesellschaft. So hilfreich dies ist, um Entwicklungen bezeichnen zu können, so sehr gilt doch zu beachten, dass es weder eine völlig säkulare, noch eine völlig disziplinierte Gesellschaft je gegeben hat. Diese Art von Idealtypen liegt nicht irgendwo im Zentrum der Streuung realer Phänomene. Es sind Fluchtpunkte von Entwicklungslinien irgendwo im Unerreichbaren, die außerhalb des Horizonts des Historischen liegen - sie sind ein Stück weit Utopie.

Mit welcher Legitimität dominieren sie den Blick unserer Analyse, die doch eigentlich der Rekonstruktion vergangener Realität verpflichtet ist? Was regelt ihre Auswahl? Und welche heuristische und darstellungstechnische Bringschuld handeln wir uns mit ihnen ein?

Will man wenigstens die zuletzt gestellte Frage knapp beantworten, muss auf den Plot der Moderne zurück gekommen werden, der in besonders ausgeprägter Weise dazu geeignet ist, den Vorgang der Zielerreichung zu dramatisieren. Gerade dort, wo nicht lediglich zielführende Kräfte herausgearbeitet werden, sondern auch die zeitgenössischen Gegenkräfte, wird zugleich der Stoff zusammengetragen, mit dem sich die Karriere der zielführenden Kraft, ihr Erscheinen, ihr Sich-Durchsetzen erst recht in Szene setzen lässt. So wird Spannung erzeugt und eine Geschichte der Widerstände und meist auch ihrer letztlichen, teils heroischen Überwindung erzählt. Eine Geschichte der Freiheit erzählt so immer auch über die Beharrungskräfte der Unfreiheit und umgekehrt. Alle Prozess- und Modernisierungsgeschichten scheinen mir so konstruiert. Sie entfalten einen Sog des Ziels, also eine sehr wesentliche, sehr mächtige Teleologie. Sie erzeugen Spannung durch Widerstände, Schwellen, Herausforderungen, und schließlich Erlösung durch Figuren der Überwindung dieser Widerstände wie auch durch das Überschreiten von Schwellen. Das Problem liegt nicht in der Vereinfachung, die damit einhergeht, denn auf Vereinfachung ist jede Art von Darstellung angewiesen. Problematisch ist der Modus der Vereinfachung, der diesen Plot der Moderne ein ums andere Mal wiederholt, der immer davon erzählt, dass sich ein Gedanke, eine Technik, ein Ideal durchsetzen möchte, auf Widerstände trifft und diese letztlich überwindet. Selbst wo wir vom Scheitern erzählen, spiegeln wir das Ideal des Erfolges. Ganz gleich wie sehr man die Moderne inhaltlich kritisiert, indem man ihren Plot zur Anwendung bringt, verspielt man die Chance, 
den Ort kritischer Distanz und eine Sprache ihrer Entlarvung zu erarbeiten. $^{31}$

So spannend der Plot also auch sein mag, er sollte keinesfalls die Agenda der zukünftigen Frühneuzeitforschung dominieren, denn damit wäre das historische Feld der Frühen Neuzeit auf die Rolle festgelegt, entweder Pioniere aufzuzählen (Protomodernität) oder die Statik und Widerständigkeit (Vormodernität) vor Augen zu führen, welche die eigentliche Moderne dann zu überwinden hat. Es gilt darüber zu reflektieren, welchen Typus von Begriffen wir forschungsleitend zum Einsatz bringen und auf welche Weise wir die historische Tiefenstruktur organisieren. Insgesamt scheint es mir dabei notwendig, die Beobachtungspräferenz von den Postulaten einer Zeit auf ihre Praktiken zu verschieben. Dies ist in Teilen der Forschung bereits vergleichsweise stillschweigend geschehen und ist nicht nur methodisch für sich genommen interessant, sondern für den strategischen Ort der Frühneuzeitforschung entscheidend. Denn sobald die Präferenz von der Ebene der Begriffe auf jene der Praktiken schwenkt, kommen wieder längere Kontinuitäten in den Blick. Lang gestreckte Entwicklungen werden sichtbar, welche durch die mächtigen Postulate des Bruches der politischen Systeme und Grundbegriffe um 1800 abgeschattet worden waren. Dazu gehören Kontinuitäten der Rechtskultur, aber auch viele Elemente der politischen und ökonomischen Verfahren, welche im Rahmen der lang gestreckten Moderne neu zu bewerten sind.

Dass mit den Praktiken zugleich abermals etwas weitaus Latenteres, weniger Explizites Gegenstand der Forschung wird als Begriffe, bringt eine neue Herausforderung in Hinsicht auf die Thematisierung von Tiefe mit sich. Dem kann, wie im Rahmen dieses Beitrages gezeigt wurde, jedoch mit der Gelassenheit einer langen, produktiven Tradition und einer daran geschulten methodischen Sensibilität entgegengesehen werden. Nun kann getrost auf die Unterstellung unsichtbarer Wesentlichkeit verzichtet werden. Und nun lässt sich mit einer offenen Agenda und mit einem Forschungsdesign arbeiten, welches die verschiedenen Schichten nicht mehr im Dienste des Plots der Zielerreichung und Überwindung gegeneinander in Stellung zu bringen hat. Weder die Kraft, noch die Gegenkraft ist in die Tiefe zu verlegen. Und Widersprüche sind nicht hinsichtlich ihrer Überwindung interessant (und in dieser Weise zu dramatisieren), sondern - ganz im Gegenteil - aufgrund ihrer historischen Persistenz.

31 Wir haben, wie Foucault das ausdrückte, „die unreflektierten Kontinuitäten außer Kurs zu setzen, durch die man im Voraus den Diskurs organisiert, den man zu analysieren vorhat", vgl. Foucault 1992, 38. 


\section{Bibliographie}

Andreas, Willy (['61932] 1959): Deutschland vor der Reformation. Eine Zeitenwende. Stuttgart: Deutsche Verlags-Anstalt.

Büttgen, Philippe (2009): „Unsichtbare Grenzen. Noch einmal zum reformatorischen Gewissensbegriff und dessen Deutung als Signatur der Neuzeit", in: Neuhaus, Helmut (Hrsg.): Die Frühe Neuzeit als Epoche. München: Oldenbourg (= Historische Zeitschrift; Beihefte, 49), 237-250.

Cellarius, Christoph ( 1696): Historia nova, hoc est XVI et XVII saeculorum qua eiusdem auctoris historiae, antiqua et mediiaevi, ad nostra tempora continenti ordine proferuntur, cum notis erpetuis et indice rerum. Halle: Henckel.

Foucault, Michel (1992): Archäologie des Wissens. Frankfurt a.M.: Suhrkamp.

Goldstone, Jack A. (1998): „The Problem of the 'Early Modern' World“, in: Journal of the Economic and Social History of the Orient 41, 249-284.

Hoffmann, Eduard (1848): Woher und Wohin? Eine populäre Schrift zur Aufklärung über die socialpolitische Bewegung der Neuzeit. Königsberg: Pfitzer \& Heilmann.

Koselleck, Reinhart (1979): „Neuzeit'. Zur Semantik moderner Bewegungsbegriffe“, in: ders. (Hrsg.): Vergangene Zukunft. Zur Semantik geschichtlicher Zeiten. Frankfurt a.M.: Suhrkamp, 300-348.

Koselleck, Reinhart (2003): „Wie neu ist die Neuzeit“, in: ders. (Hrsg.): Zeitschichten. Studien zur Historik. Mit einem Beitrag von Hans-Georg Gadamer. Frankfurt a.M.: Suhrkamp, 225-239.

Kracauer, Siegfried (1993): Von Caligari zu Hitler. Eine psychologische Geschichte des deutschen Films. Frankfurt a.M.: Suhrkamp.

Luther, Martin (1912-1921): Tischreden. Kritische Gesamtausgabe. Bd. 1. Weimar: Böhlau.

Marggraf, Hermann (Hrsg.) (1847): Politische Gedichte aus Deutschlands Neuzeit. Von Klopstock bis auf die Gegenwart. Leipzig: Kößling.

Menzel, Wolfgang (1871): Die wichtigsten Weltbegebenheiten vom Prager Frieden bis zum Kriege mit Frankreich (1866-1870). Bd. 1. Stuttgart: Krabbe.

Mout, Nicolette (1989): „Einleitung“, in: Oestreich, Gerhard: Antiker Geist und moderner Staat bei Justus Lipsius (1547-1606). Der Neustoizismus als politische Bewegung. Hrsg. u. eingel. von Nicolette Mout. Göttingen: Vandenhoeck \& Ruprecht (= Schriftenreihe der Historischen Kommission bei der Bayerischen Akademie der Wissenschaften, 38), 11-35.

Müller, Jan-Dirk (1991): „Alt' und 'Neu' in der Epochenerfahrung um 1500. Ansätze zur kulturgeschichtlichen Periodisierung in frühneuhochdeutschen Texten“, in: Haug, Walter/Wachinger, Burghart (Hrsg.): Traditionswandel und Traditionsverhalten. Tübingen: Niemeyer (= Fortuna vitrea, 5), $121-$ 144.

Münch, Paul (1984): Ordnung, Fleiß und Sparsamkeit. Texte und Dokumente zur Entstehung der bürgerlichen Tugenden. München: Deutscher Taschenbuch Verlag (= dtv-Dokumente, 2940).

Oestreich, Gerhard (1989): Antiker Geist und moderner Staat bei Justus Lipsius (1547-1606). Der Neustoizismus als politische Bewegung. Hrsg. u. eingel. von Nicolette Mout. Göttingen: Vandenhoeck \& Ruprecht (= Schriftenreihe der 
Historischen Kommission bei der Bayerischen Akademie der Wissenschaften, 38).

Pot, Johan Hendrik van der (1999): Sinndeutung und Periodisierung der Geschichte. Eine systematische Übersicht der Theorien und Auffassungen. Leiden/Boston / Köln: Brill.

Ranke, Leopold von (1975): „Vorlesung über die Neuere Geschichte, Winter 1833-1834. Nachschrift von Siegfried Hirsch“, in: Dotterweich, Volker/Fuchs, Walther Peter (Hrsg.): Aus Werk und Nachlass. Bd. 4: Vorlesungseinleitungen. München/Wien: Oldenbourg, $104 \mathrm{f}$.

Reinhard, Wolfgang (1993): „Historiker, 'Modernisierung' und Modernisierung. Erfahrungen mit dem Konzept 'Modernisierung' in der neueren Geschichte“, in: Haug, Walter / Wachinger, Burghart (Hrsg.): Innovation und Originalität. Tübingen: Niemeyer (= Fortuna vitrea, 9), 53-69.

Rexroth, Frank (2008): „Das Mittelalter und die Moderne in den Meistererzählungen der historischen Wissenschaften", in: Zeitschrift für Literaturwissenschaft und Linguistik 151, 12-31.

Schorn-Schütte, Luise (1999a): „Altprotestantismus und moderne Welt. Ernst Troeltschs 'liberale' Deutungsmuster der nachreformatorischen Geschichte“, in: dies. (Hrsg.): Alteuropa oder Frühe Moderne? Deutungsmuster für das 16. bis 18. Jahrhundert aus dem Krisenbewußtsein der Weimarer Republik in Theologie, Rechts-und Geschichtswissenschaft. Berlin: Duncker \& Humblot (= Zeitschrift für Historische Forschung; Beiheft, 23), 45-54.

Schorn-Schütte, Luise (Hrsg.) (1999b): Alteuropa oder Frühe Moderne? Deutungsmuster für das 16. bis 18. Jahrhundert aus dem Krisenbewußtsein der Weimarer Republik in Theologie, Rechts- und Geschichtswissenschaft. Berlin: Duncker \& Humblot (= Zeitschrift für Historische Forschung; Beiheft, 23).

Schulze, Winfried (1990): „Ende der Moderne? Zur Korrektur unseres Begriffs der Moderne aus historischer Sicht", in: Meier, Heinrich (Hrsg.): Zur Diagnose der Moderne. München/Zürich: Piper (= Veröffentlichung der Carl Friedrich von Siemens Stiftung, 3), 69-97.

Schulze, Winfried (1993): „'Von den großen Anfängen des neuen Welttheaters'. Entwicklung, neue Ansätze und Aufgaben der Frühneuzeitforschung", in: Geschichte in Wissenschaft und Unterricht 44/12, 3-18.

Skalweit, Stephan (1987): Gestalten und Probleme der frühen Neuzeit. Ausgewählte Aufsätze. Berlin: Duncker \& Humblot (= Historische Forschungen, 32).

Tönnies, Ferdinand (1998): „Geist der Neuzeit“, in: ders. (Hrsg.): Gesamtausgabe. Bd. 22: 1932-1936: Geist der Neuzeit, Schriften, Rezensionen. Berlin/New York: De Gruyter, $1-223$.

Tschopp, Silvia Serena (2005): „Das Unsichtbare begreifen. Die Rekonstruktion historischer Wahrnehmungsmodi als methodische Herausforderung der Kulturgeschichte", in: Historische Zeitschrift 280, 39-81.

Wagner, Peter (2009): Moderne als Erfahrung und Interpretation. Eine neue Soziologie zur Moderne. Konstanz: UVK Verlags-Gesellschaft.

Weber, Max (1920): „Zwischenbetrachtung“, in: ders.: Gesammelte Aufsätze zur Religionssoziologie. Bd. 1. Tübingen: Mohr Siebeck, 536-573.

White, Hayden (1994): Metahistory. Die historische Einbildungskraft im 19. Jahrhundert in Europa. Frankfurt a.M.: Fischer (= Fischer Wissenschaft, 11701). 
Worstbrock, Franz Josef (1974): „Über das geschichtliche Selbstverständnis des deutschen Humanismus“, in: Müller-Seidel, Walter (Hrsg.): Historizität in Sprach- und Literaturwissenschaft. Vorträge und Berichte der Stuttgarter Germanistentagung 1972. München: Fink, 499-519.

Zwierlein, Cornel (2009): „Vom Anfang und vom Ende der Frühen Neuzeit-oder: Brannte Konstantinopel 1870 in der Moderne und London 1666 in der Frühen Neuzeit?", in: Mitteilungen des SFB 573 'Pluralisierung \& Autorität in der Frühen Neuzeit' 1, 22-31.

Zwierlein, Cornel (2011): Der gezähmte Prometheus. Zum Verhältnis von Sicherheit und Moderne am Beispiel von Brandgefahr und Feuerversicherung, 1680-1850. Göttingen: Vandenhoeck \& Ruprecht (= Umwelt und Gesellschaft, 3). 
